\title{
Synchronization of Chaotic Gyros Based on Robust Nonlinear Dynamic Inversion
}

\author{
Inseok Yang and Dongik Lee \\ School of Electronics Engineering, Kyungpook National University, Daegu 702-701, Republic of Korea \\ Correspondence should be addressed to Dongik Lee; dilee@ee.knu.ac.kr
}

Received 4 February 2013; Accepted 12 June 2013

Academic Editor: Carlos J. S. Alves

Copyright (C) 2013 I. Yang and D. Lee. This is an open access article distributed under the Creative Commons Attribution License, which permits unrestricted use, distribution, and reproduction in any medium, provided the original work is properly cited.

\begin{abstract}
A robust nonlinear dynamic inversion (RNDI) technique is proposed in order to synchronize the behavior of chaotic gyros subjected to uncertainties such as model mismatches and disturbances. Gyro is a crucial device that measures and maintains the orientation of a vehicle. By Leipnik and Newton in 1981, chaotic behavior of a gyro under specific conditions was established. Hence, controlling and synchronizing a gyro that shows irregular (chaotic) motion are very important. The proposed synchronization method is based on nonlinear dynamic inversion (NDI) control. NDI is a nonlinear control technique that removes the original system dynamics into the user-defined desired dynamics. Since NDI removes the original dynamics directly, it does not need linearizing and designing gain-scheduled controllers for each equilibrium point. However, achieving perfect cancellation of the original nonlinear dynamics is impossible in real applications due to model uncertainties and disturbances. This paper proposes the robustness assurance method of NDI based on sliding mode control (SMC). Firstly, similarities of the conventional NDI control and SMC are provided. And then the RNDI control technique is proposed. The feasibility and effectiveness of the proposed method are demonstrated by numerical simulations.
\end{abstract}

\section{Introduction}

Chaotic behavior is a widely observed phenomenon in nature as well as nonlinear systems. This presents a challenging problem as direct control of chaotic systems is very difficult. Chaos synchronization is a control process in which the motions of coupled chaotic systems under different initial conditions are synchronized [1-10]. Since chaotic systems are very sensitive to the initial conditions, system behaviors initiated distinctly cause irregular different motions. Figure 1 shows the irregular motions of two chaotic systems, called drive and response systems, under different initial conditions. Since the work of Pecora and Carroll in 1990, various chaos synchronization techniques based on nonlinear control theory have been developed. As shown in Figure 2, chaos synchronization of two chaotic systems entails controlling the motion of the response system by introducing an additional control input, such as adaptive control [2], passive control [3], backstepping control $[4,5]$, and sliding mode control $[6,7]$. The feasibility of controlling chaotic systems has been explored in various fields of science and engineering, such as secure communications, chemical reactions, power converters, biological systems, and information processing $[2,5-10]$.

In this paper, a robust nonlinear dynamic inversion (RNDI) technique is proposed for the synchronization of chaotic gyros perturbed by bounded uncertainties. Gyro is a device that measures and maintains the orientation of a vehicle. For this reason, gyro is one of the most crucial instruments in safety-critical systems such as aircrafts, spacecrafts, and underwater vehicles. Since the work of Leipnik and Newton [11] who verified chaotic behavior of gyro under specific conditions in 1981, controlling and synchronizing a gyro has received considerable attention $[5,6,10]$. However, synchronization of gyros showing irregular (chaotic) motions is still a challenging problem.

The proposed RNDI control method is an extension of nonlinear dynamic inversion (NDI). NDI is a nonlinear control technique that removes the original system dynamics into the user-defined desired dynamics [12]. Different from many other nonlinear controllers that linearize the original system in order to design gain-scheduled controllers for 


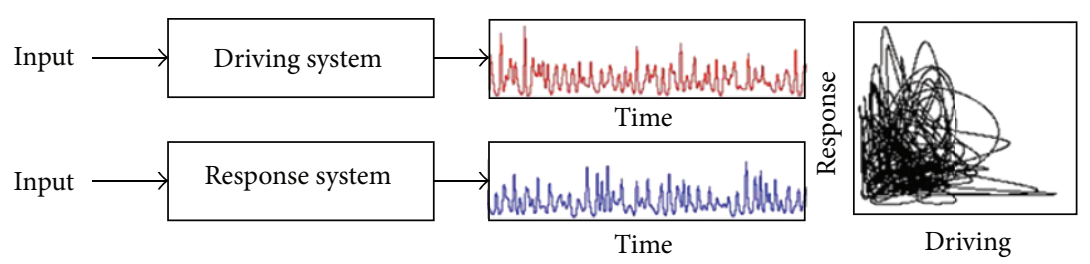

FIGURE 1: Behaviors of unsynchronized chaotic systems.

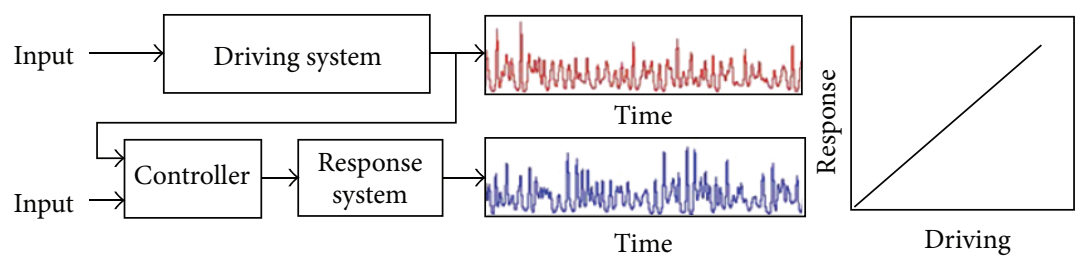

FIGURE 2: Behaviors of synchronized chaotic systems.

each equilibrium points, NDI does not need designing gainscheduled controllers, since it removes the system original dynamics directly. Hence, NDI avoids difficulties of ensuring stability between operating points. For this reason, NDI is widely applied in the aerospace industry [13-16] that operates in various equilibrium points. However, the main drawback of NDI is poor robustness. That is, achieving perfect cancellation of original nonlinear dynamics is impossible in real applications due to the presence of model uncertainties and disturbances acting on the system. Hence, the robustness issue has received a great deal of interest in designing NDI [12-16]. One of the most widely used methods for solving this issue is employing an additional linear controller to form an outer-loop controller while NDI works as an inner-loop controller. However, the main drawback of this method is increased order of the control system. For instance, the controller order increases to 14 when using an $H_{\infty}$-based outerloop controller for X-38 [12]. Recently, Yang et al. proposed the robust dynamic inversion (RDI) control method for linearized systems. The proposed RDI controller guarantees stability against uncertainties without using any outer-loop controller [14]. This paper proposes an extension of the RDI controller for nonlinear systems. Similarities of the NDI and the SMC are provided firstly. And then the RNDI control technique is proposed by following the design method of the SMC provided in [17]. Numerical simulations of the synchronization problem with chaotic gyros show that the proposed method achieves the desired control performance, although the systems are perturbed by uncertainties.

\section{The Proposed Robust Nonlinear Dynamic Inversion}

2.1. Conceptual Design of Nonlinear Dynamic Inversion. Let us consider the following nonlinear dynamical system:

$$
\begin{gathered}
\dot{x}=f(x)+g(x) u, \\
y=h(x),
\end{gathered}
$$

where $x \in \mathbf{X}$, an open set of $R^{n}$, is a state vector and $u$ : $R \rightarrow R$ is an input. $f$ and $g$ defined on $\mathbf{X}$ are a smooth nonlinear state dynamic function and a smooth nonlinear control distribution function, respectively. Further, $y \in \mathbf{Y}$, an open set of $R$, is an output and $h: R^{n} \rightarrow R$ is a smooth nonlinear function. If $L_{g} h(x) \neq 0$ for all $x \in \mathbf{X}$, then the NDI control input for a single-input and single-output (SISO) system is designed as follows:

$$
u_{\mathrm{NDI}}=\left[L_{g} h(x)\right]^{-1}\left[\dot{y}_{\mathrm{des}}-L_{f} h(x)\right]
$$

where $L_{(\cdot)}$ is the Lie derivative with respect to (.) and $y_{\text {des }}$ represents the desired dynamics that determines the system response after canceling the original dynamics. By substituting (2) into (1), the dynamic system controlled by NDI can be represented as follows:

$$
\dot{y}=\dot{y}_{\mathrm{des}}
$$

Hence, the original nonlinear dynamics are replaced with the desired dynamics. However, the robustness issue must be considered in designing an NDI controller as perfect cancelation of the original dynamics cannot be achieved in real applications.

2.2. The Proposed Robust Nonlinear Dynamic Inversion. Let $\sigma: \mathbf{Y} \rightarrow R$ be a smooth function such that $\{y \mid \sigma(y)=0\}$ is a smooth manifold. In general, $\sigma(y)=0$ is known as a sliding surface or sliding manifold in sliding mode control (SMC) theory $[17,18]$. SMC consists of two phases called reaching and sliding phases [18]. One method for designing a sliding surface is using equivalent control. This method, defined as ideal sliding motion, determines an equivalent input, $u_{\text {eq }}$, that forces the output to stay on the sliding surface $\sigma(y)=0$. 
In this approach, the equivalent input can be analyzed by means of the manifold invariant conditions [17]:

$$
\begin{aligned}
& \dot{\sigma}(y)=\dot{\sigma}(h(x))=\left(\frac{\partial \sigma}{\partial y}\right)\left[L_{f} h(x)+L_{g} h(x) u_{\mathrm{eq}}\right]=0, \\
& \Longrightarrow u_{\mathrm{eq}}=-\left[\left(\frac{\partial \sigma}{\partial y}\right) L_{g} h(x)\right]^{-1}\left[\left(\frac{\partial \sigma}{\partial y}\right) L_{f} h(x)\right],
\end{aligned}
$$

where $\left[(\partial \sigma / \partial y) L_{g} h(x)\right]$ is assumed to be nonsingular. Then, the dynamics of the system on the sliding surface is governed by

$$
\dot{y}=\left[I-L_{g} h(x)\left[\left(\frac{\partial \sigma}{\partial y}\right) L_{g} h(x)\right]^{-1}\left(\frac{\partial \sigma}{\partial y}\right)\right] L_{f} h(x) .
$$

The dynamics (5) is defined as the ideal sliding dynamics. As shown in (5), the characteristics of the ideal sliding dynamics are determined by the sliding surface. Hence, choosing a sliding surface that makes the system stable is critical in the design of a sliding mode controller.

For the desired dynamics, $y_{\text {des }} \in \mathbf{Y}$, let $y^{*}=y_{\text {des }}-y=$ $y_{\text {des }}-h(x) \in \mathbf{Y}$. Then, the equivalent input can be obtained as follows:

$$
\begin{aligned}
\dot{\sigma}\left(y^{*}\right)= & \left(\frac{\partial \sigma}{\partial y^{*}}\right) \dot{y}^{*}=\left(\frac{\partial \sigma}{\partial y^{*}}\right) \dot{y}_{\mathrm{des}} \\
& -\left(\frac{\partial \sigma}{\partial y^{*}}\right)\left[L_{f} h(x)+L_{g} h(x) u_{\mathrm{eq}}\right]=0, \\
\Longrightarrow u_{\mathrm{eq}}= & {\left[\left(\frac{\partial \sigma}{\partial y^{*}}\right) L_{g} h(x)\right]^{-1} } \\
& \times\left[\left(\frac{\partial \sigma}{\partial y^{*}}\right) \dot{y}_{\mathrm{des}}-\left(\frac{\partial \sigma}{\partial y^{*}}\right) L_{f} h(x)\right] .
\end{aligned}
$$

In (6), if the sliding surface is identity (i.e., $\sigma\left(y^{*}\right)=y^{*}$ ), then the equivalent input yields

$$
u_{\mathrm{eq}}=\left[L_{g} h(x)\right]^{-1}\left[\dot{y}_{\mathrm{des}}-L_{f} h(x)\right] .
$$

Comparing (7) with (2), the equivalent input designed with an identity sliding surface can be considered as the conventional NDI input.

Definition 1. Let $\sigma_{\mathrm{NDI}}: \mathbf{Y} \rightarrow R$ be a smooth function. Then $\sigma_{\mathrm{NDI}}=0$ is defined as the NDI surface if $\sigma_{\mathrm{NDI}}(y)=y$. That is, if the sliding surface is identity, then the sliding surface is defined as the NDI surface.

As the NDI input is represented as the equivalent input of the identity sliding surface, it controls the output on the NDI surface. Moreover, since the error between the desired dynamics and output is forced to be zero by the NDI input, the output is driven by the desired dynamics.

Theorem 2. The NDI law is well defined if and only if $L_{g} h(x) \neq 0$, for all $x \in \mathbf{X}$.
Proof. Suppose that the NDI law is well defined; that is, $u_{\mathrm{NDI}}$ exists uniquely. Then, it is clear that $L_{g} h(x) \neq 0$ by (7). Because if $L_{g} h(x)=0$, then $\dot{y}_{\text {des }}-L_{f} h(x)$ has to be zero. However, it is impossible to achieve the system $\dot{y}_{\text {des }}-$ $L_{f} h(x)=0$ in real applications. Moreover, if $\dot{y}_{\text {des }}-L_{f} h(x)=$ 0 , then the NDI input exists trivially. Thus, it contradicts the hypothesis of uniqueness. Hence, if the NDI law is well defined, then $L_{g} h(x) \neq 0$.

Conversely, suppose that $L_{g} h(x) \neq 0$. Then, from (7), $u_{\mathrm{NDI}}$ exists. To prove its uniqueness, it is assumed that $u_{\mathrm{NDI}, 1}$ and $u_{\mathrm{NDI}, 2}$ are two distinct NDI inputs. From the manifold invariant condition, $\dot{y}_{\text {des }}-L_{f} h(x)-L_{g} h(x) u_{\mathrm{NDI}, 1}=0=\dot{y}_{\text {des }}-$ $L_{f} h(x)-L_{g} h(x) u_{\mathrm{NDI}, 2}$. Hence, $\left[L_{g} h(x)\right]\left(u_{\mathrm{NDI}, 1}-u_{\mathrm{NDI}, 2}\right)=0$. Since $L_{g} h(x) \neq 0$, then $u_{\mathrm{NDI}, 1}=u_{\mathrm{NDI}, 2}$. This contradicts the condition of $u_{\mathrm{NDI}, 1} \neq u_{\mathrm{NDI}, 2}$. Thus, if $L_{g} h(x) \neq 0$, then the NDI law is well defined.

Hence, the NDI law is well defined if and only if $L_{g} h(x) \neq 0$, for all $x \in \mathbf{X}$.

The proposed robust nonlinear dynamic inversion law, $u$, can be obtained by taking the extreme control values [17]:

$$
u= \begin{cases}u^{+}, & \text {if } y<y_{\text {des }} \\ u^{-}, & \text {if } y>y_{\text {des }}\end{cases}
$$

where $u^{+} \neq u^{-}$. The following is then satisfied:

$$
\begin{aligned}
& \lim _{h(x) \rightarrow y_{\mathrm{des}}^{+}} L_{f+g u^{+}} h(x)>\dot{y}_{\mathrm{des}}, \\
& \lim _{h(x) \rightarrow y_{\mathrm{des}}^{-}} L_{f+g u^{-}} h(x)<\dot{y}_{\mathrm{des}} .
\end{aligned}
$$

Then, the point of the controlled vector fields $L_{f+q u^{+}} h$ and $L_{f+g u^{u}} h$ moves toward the NDI surface. Figure 3 illustrates the robust nonlinear dynamics inversion regime on the NDI surface.

Theorem 3. If $u^{+}>u^{-}$, then $L_{g} h(x)>0$ and, conversely, if $u^{+}<u^{-}$, then $L_{g} h(x)<0$.

Proof. It is clear directly from (9). Since $L_{f} h(x)+L_{g} h(x) u^{+}>$ $\dot{y}_{\text {des }}$ and $L_{f} h(x)+L_{g} h(x) u^{-}<\dot{y}_{\text {des }}$, then $L_{g} h(x)\left(u^{+}-u^{-}\right)>0$. Hence, if $u^{+}>u^{-}$, then $L_{g} h(x)>0$ and, conversely, if $u^{+}<$ $u^{-}$, then $L_{g} h(x)<0$.

\section{Theorem 4. The RNDI law exists locally if and only if}

$$
\min \left\{u^{-}, u^{+}\right\}<u_{N D I}<\max \left\{u^{-}, u^{+}\right\} .
$$

Proof. Suppose that the NDI law exists and $u^{+}>u^{-}$, that is, $u^{+}=\max \left\{u^{-}, u^{+}\right\}$and $u^{-}=\min \left\{u^{-}, u^{+}\right\}$. From (2) and (9), $L_{f} h(x)+L_{g} h(x) u^{+}>\dot{y}_{\mathrm{des}} \Rightarrow L_{g} h(x) u^{+}>\dot{y}_{\mathrm{des}}-L_{f} h(x)=$ $L_{g} h(x) u_{\mathrm{NDI}}$. Since $u^{+}>u^{-}$, by Theorem $3, L_{g} h(x)>0$. Hence, $u^{+}>u_{\mathrm{NDI}}$. Similarly, $L_{f} h(x)+L_{g} h(x) u^{-}<\dot{y}_{\text {des }} \Rightarrow$ $L_{g} h(x) u^{-}<\dot{y}_{\mathrm{des}}-L_{f} h(x)=L_{g} h(x) u_{\mathrm{NDI}}$. Since $u^{+}<u^{-}$, $L_{g} h(x)>0$. Hence, $\min \left\{u^{-}, u^{+}\right\}=u^{-}<u_{\mathrm{NDI}}(x)<u^{+}=$ $\max \left\{u^{-}, u^{+}\right\}$. 


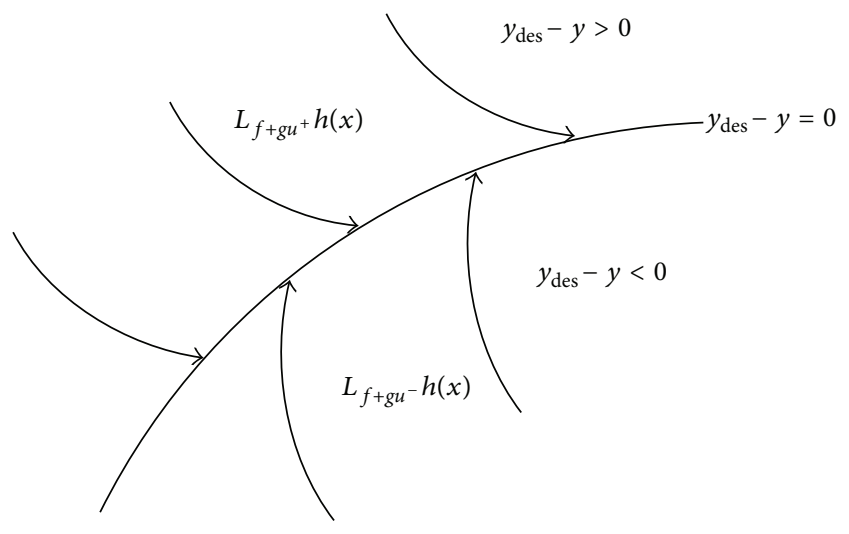

FIGURE 3: The nonlinear dynamic inversion regime on the NDI surface.

Conversely, if $u^{+}<u^{-}$, then $u^{-}=\max \left\{u^{-}, u^{+}\right\}$and $u^{+}=\min \left\{u^{-}, u^{+}\right\}$. From (9), $L_{f} h(x)+L_{g} h(x) u^{-}<\dot{y}_{\text {des }} \Rightarrow$ $L_{g} h(x) u^{-}<\dot{y}_{\text {des }}-L_{f} h(x)=L_{g} h(x) u_{\mathrm{NDI}}$. Since $u^{-}>u^{+}$, by Theorem 3, $L_{g} h(x)<0$. Hence, $u^{-}>u_{\mathrm{NDI}} \Rightarrow \max \left\{u^{-}, u^{+}\right\}>$ $u_{\mathrm{NDI}}$. Similarly, $L_{f} h(x)+L_{g} h(x) u^{+}>\dot{y}_{\text {des }} \Rightarrow L_{g} h(x) u^{+}>$ $\dot{y}_{\text {des }}-L_{f} h(x)=L_{g} h(x) u_{\mathrm{NDI}}$. Since $L_{g} h(x)<0, u^{+}<u_{\mathrm{NDI}}$. Thus, $\min \left\{u^{-}, u^{+}\right\}<u_{\mathrm{NDI}}$. Hence, $\min \left\{u^{-}, u^{+}\right\}<u_{\mathrm{NDI}}<$ $\max \left\{u^{-}, u^{+}\right\}$.

Conversely, suppose that $u_{\mathrm{NDI}}$ is a smooth feedback function satisfying (2) and (10). Then, we have

$$
0<u_{\mathrm{NDI}}-\min \left\{u^{-}, u^{+}\right\}<\max \left\{u^{-}, u^{+}\right\}-\min \left\{u^{-}, u^{+}\right\} .
$$

Simply, set $u^{-}=\min \left\{u^{-}, u^{+}\right\}$and $u^{+}=\max \left\{u^{-}, u^{+}\right\}$. Then (11) is converted as follows:

$$
0<u_{\mathrm{NDI}}-u^{-}<u^{+}-u^{-} \Longrightarrow 0<w_{\mathrm{NDI}}:=\frac{u_{\mathrm{NDI}}-u^{-}}{u^{+}-u^{-}}<1 .
$$

This leads to $0<1-w_{\mathrm{NDI}}<1$. Moreover, the following relationships are satisfied:

$$
\begin{aligned}
\dot{y}_{\mathrm{des}}- & \left(L_{f} h(x)+L_{g} h(x) u_{\mathrm{NDI}}\right) \\
= & w_{\mathrm{NDI}}\left\{\dot{y}_{\mathrm{des}}-\left(L_{f} h(x)+L_{g} h(x) u^{+}\right)\right\} \\
& +\left(1-w_{\mathrm{NDI}}\right)\left\{\dot{y}_{\mathrm{des}}-\left(L_{f} h(x)+L_{g} h(x) u^{-}\right)\right\}=0 .
\end{aligned}
$$

From (13), $\dot{y}_{\text {des }}-L_{f} h(x)-L_{g} h(x) u^{+}$and $\dot{y}_{\text {des }}-L_{f} h(x)-$ $L_{g} h(x) u^{-}$have opposite signs. Since the orientation of NDI is arbitrary, it is reasonable that $L_{f} h(x)+L_{g} h(x) u^{-}<\dot{y}_{\text {des }}$ and $L_{f} h(x)+L_{g} h(x) u^{+}>\dot{y}_{\text {des }}$. Thus, (9) is satisfied. Hence, if the control law $u_{\mathrm{NDI}}$ satisfies the condition of (10), then the RNDI law exists.

Corollary 5. The RNDI law exists locally if the RNDI law is designed as

$$
u_{R N D I}=u_{N D I}+k\left[L_{g} h(x)\right]^{-1} \operatorname{sgn}\left(y_{d e s}-h(x)\right), \quad k>0 .
$$

Proof. If $y_{\mathrm{des}}-y>0$, then $u_{\mathrm{RNDI}}=u_{\mathrm{NDI}}+k\left[L_{g} h(x)\right]^{-1} \equiv u^{+}$. If $y_{\mathrm{des}}-y<0$, then $u_{\mathrm{RNDI}}=u_{\mathrm{NDI}}-k\left[L_{g} h(x)\right]^{-1} \equiv u^{-}$. Thus, $\min \left\{u_{\mathrm{NDI}}-k\left[L_{g} h(x)\right]^{-1}, u_{\mathrm{NDI}}+k\left[L_{g} h(x)\right]^{-1}\right\}<u_{\mathrm{NDI}}<$ $\max \left\{u_{\mathrm{NDI}}-k\left[L_{g} h(x)\right]^{-1}, u_{\mathrm{NDI}}+k\left[L_{g} h(x)\right]^{-1}\right\}$. Hence, by Theorem 4 , the RNDI law exists locally.

Stability of the closed-loop system designed by the proposed RNDI control law is proven by a Lyapunov stability criterion.

Theorem 6. Consider the following system:

$$
\begin{gathered}
\dot{x}(t)=f(x(t))+g(x(t)) u(t)+d_{X}(t), \\
y(t)=h(x(t))+d_{Y}(t),
\end{gathered}
$$

where $d_{X}(t) \in R^{n}$ and $d_{Y}(t) \in R$ are bounded disturbances. For $\xi(t) \in R$ such that $\xi(t)=(\partial h / \partial x) d_{X}(t)+\dot{d}_{Y}(t)$, if $|\xi(t)|<k$ for the positive switching gain $k$; then the system (15) designed by the proposed RNDI control law (14) is globally stable.

Proof. Suppose that $y^{*}=y_{\mathrm{des}}-y$. To prove the stability of the closed-loop system designed by the proposed RNDI control law, a Lyapunov stability criterion is considered. Choose a Lyapunov candidate as

$$
V\left(y^{*}(t)\right)=\frac{1}{2} y^{*}(t) y^{*}(t)
$$

Then, the derivative of $V\left(y^{*}(t)\right)$ yields

$$
\begin{aligned}
& \dot{V}\left(y^{*}(t)\right)=y^{*}(t) \dot{y}^{*}(t) \\
& =y^{*}(t)\left[\dot{y}_{\mathrm{des}}(t)-\dot{y}(t)\right] \\
& =y^{*}(t)\left[\dot{y}_{\mathrm{des}}(t)\right. \\
& -\left\{L_{f} h(x)+L_{g} h(x) u_{\mathrm{RNDI}}\right. \\
& \left.\left.+\left(\frac{\partial h}{\partial x}\right) d_{X}(t)+\dot{d}_{Y}(t)\right\}\right] \\
& =y^{*}(t)\left[\dot{y}_{\mathrm{des}}(t)\right. \\
& -\left\{L_{f} h(x)+L_{g} h(x)\right. \\
& \times\left\{u_{\mathrm{NDI}}+k\left[L_{g} h(x)\right]^{-1}\right. \\
& \left.\left.\left.\times \operatorname{sgn}\left(y^{*}(t)\right)\right\}+\xi(t)\right\}\right] \\
& =y^{*}(t)\left[\dot{y}_{\mathrm{des}}(t)\right. \\
& -\left\{L_{f} h(x)+L_{g} h(x) u_{\mathrm{NDI}}\right. \\
& \left.\left.+k \operatorname{sgn}\left(y^{*}(t)\right)+\xi(t)\right\}\right] \\
& =y^{*}(t)\left[-k \operatorname{sgn}\left(y^{*}(t)\right)-\xi(t)\right]
\end{aligned}
$$




$$
\begin{aligned}
= & {\left[y_{\text {des }}(t)-y(t)\right] } \\
& \times\left[-k \operatorname{sgn}\left(y_{\text {des }}(t)-y(t)\right)-\xi(t)\right] \\
\leq & |\xi(t)|\left|y_{\text {des }}(t)-y(t)\right| \\
& -k\left(y_{\text {des }}(t)-y(t)\right) \operatorname{sgn}\left(y_{\text {des }}(t)-y(t)\right) \\
= & |\xi(t)|\left|y_{\text {des }}(t)-y(t)\right|-k\left|y_{\text {des }}(t)-y(t)\right| \\
= & (|\xi(t)|-k)\left|y_{\text {des }}(t)-y(t)\right| \\
< & 0 .
\end{aligned}
$$

Hence, the system controlled by the proposed RNDI controller is globally stable.

\section{Simulation Results}

In this section, the performance of the proposed robust nonlinear dynamic inversion technique is evaluated with application to the synchronization of chaotic gyros.

3.1. Dynamics of Chaotic Gyros. The dynamic equation of chaotic gyros with linear-plus-cubic damping mounted on a vibrating platform (Figure 4) can be represented as follows [10]:

$$
\begin{aligned}
\ddot{\theta}+\alpha^{2} & \frac{(1-\cos \theta)^{2}}{\sin ^{3} \theta}-\beta \sin \theta \\
& +c_{1} \dot{\theta}+c_{2} \dot{\theta}^{3}=f \sin \omega t \sin \theta,
\end{aligned}
$$

where $c_{1} \dot{\theta}$ and $c_{2} \dot{\theta}^{3}$ are linear and nonlinear damping terms, respectively. Further, $\alpha^{2}\left[(1-\cos \theta)^{2} / \sin ^{3} \theta\right]-\beta \sin \theta$ is a nonlinear resilience force and $f \sin \omega t$ is a parametric excitation.

By letting $x_{11}=\theta, x_{12}=\dot{\theta}$, and $g(\theta)=-\alpha^{2}[(1-$ $\left.\cos \theta)^{2} / \sin ^{3} \theta\right]$, the state equation of chaotic gyros can be transformed into the following normal form:

$$
\begin{gathered}
\dot{x}_{11}=x_{12}, \\
\dot{x}_{12}=g\left(x_{11}\right)-c_{1} x_{12}-c_{2} x_{12}^{3}+(\beta+f \sin \omega t) \sin x_{11}, \\
y_{11}=x_{11} .
\end{gathered}
$$

It is well known that for particular values of $\alpha^{2}=100, \beta=1$, $c_{1}=0.5, c_{2}=0.05, \omega=2$, and $f=35.5$, the gyro system exhibits chaotic behavior [11]. Figures 5 and 6 illustrate the irregular motion of the states and the phase portrait under the initial condition of $\left[x_{11}(0), x_{12}(0)\right]=[1,-1]$, respectively. Let $x_{1}=\left[x_{11}, x_{12}\right]^{T}$ and $x_{2}=\left[x_{21}, x_{22}\right]^{T}$ denote the states of the drive and response systems, respectively. Then, the dynamic equations of the drive and response systems with model mismatches and disturbances are represented as follows.

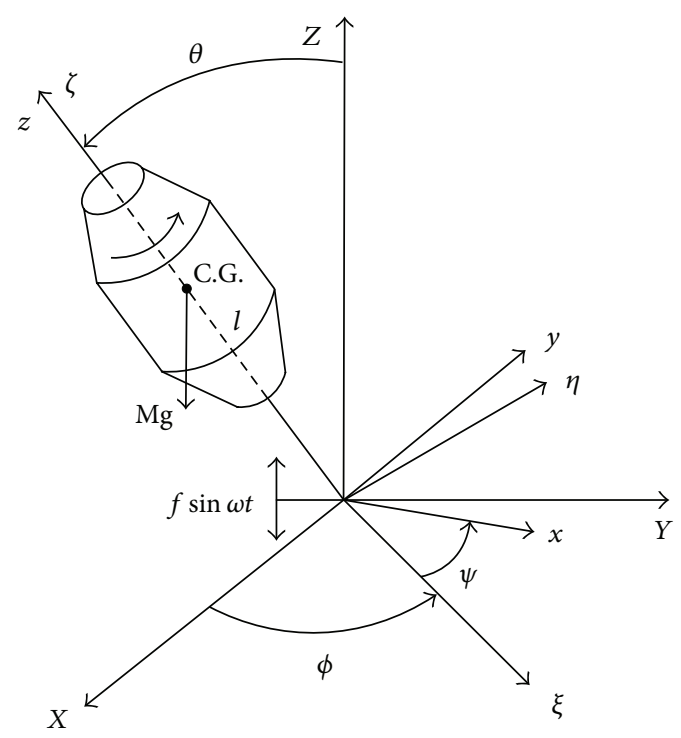

FIGURE 4: Schematic diagram of a symmetric gyroscope [10].

Drive system

$$
\begin{gathered}
\dot{x}_{11}=x_{12}, \\
\dot{x}_{12}=g\left(x_{11}\right)-c_{1} x_{12}-c_{2} x_{12}^{3}+(\beta+f \sin \omega t) \sin x_{11} \\
+\Delta f\left(x_{11}, x_{12}\right)+d_{x_{1}}(t), \\
y_{11}=x_{11},
\end{gathered}
$$

where $\Delta f\left(x_{11}, x_{12}\right)$ and $d_{x_{1}}(t)$ are assumed to be bounded model mismatches and bounded disturbance, respectively.

Response system

$$
\begin{gathered}
\dot{x}_{21}=x_{22}, \\
\dot{x}_{22}=g\left(x_{21}\right)-c_{1} x_{22}-c_{2} x_{22}^{3}+(\beta+f \sin \omega t) \sin x_{21} \\
+\Delta f\left(x_{21}, x_{22}\right)+d_{x_{2}}(t)+u(t), \\
y_{21}=x_{21},
\end{gathered}
$$

where $\Delta f\left(x_{21}, x_{22}\right)$ and $d_{x_{2}}(t)$ also denote model mismatches and disturbance, respectively, and are also assumed to be bounded. $u(t)$ is the control input provided by the proposed RNDI controller to synchronize the chaotic gyros.

If the errors between the states of the drive and response systems are defined as $e_{x_{i}}=x_{2 i}-x_{1 i}$ and $e_{y_{i}}=y_{2 i}-y_{1 i}$, then the error dynamics can be represented as follows:

$$
\begin{gathered}
\dot{e}_{x_{1}}=e_{x_{2}}, \\
\dot{e}_{x_{2}}=g\left(x_{11}, x_{21}\right)-c_{1} e_{x_{2}}-c_{2}\left(x_{22}^{3}-x_{12}^{3}\right), \\
+(\beta+f \sin \omega t)\left(\sin x_{21}-\sin x_{11}\right) \\
+\Delta f\left(x_{1}, x_{2}\right)+d(t)+u(t), \\
e_{y_{1}}=y_{21}-y_{11},
\end{gathered}
$$




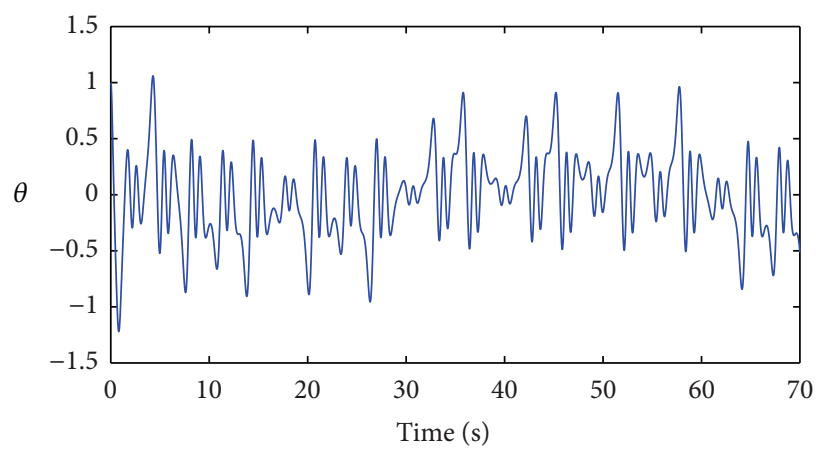

(a)

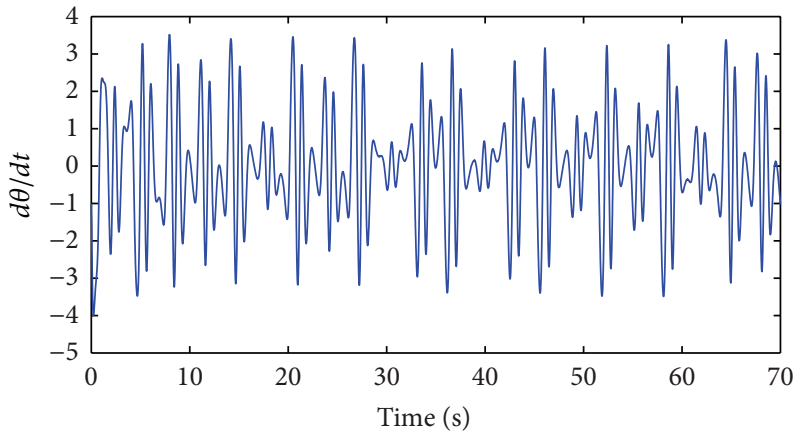

(b)

FIgURE 5: Behaviors of the chaotic gyros: (a) trajectories of $\theta$ of the chaotic gyro, (b) trajectories of $\dot{\theta}$ of the chaotic gyro.

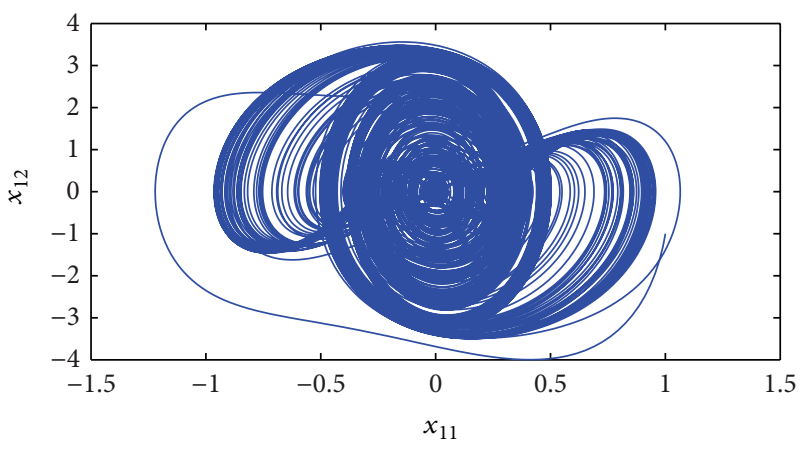

FIGURE 6: Phase portrait of the chaotic gyro.

where $g\left(x_{11}, x_{21}\right)=g\left(x_{21}\right)-g\left(x_{11}\right), \Delta f\left(x_{1}, x_{2}\right)=$ $\Delta f\left(x_{21}, x_{22}\right)-\Delta f\left(x_{11}, x_{12}\right)$, and $d(t)=d_{x_{2}}(t)-d_{x_{1}}(t)$. Since $\Delta f\left(x_{11}, x_{12}\right), \Delta f\left(x_{21}, x_{22}\right), d_{x_{1}}(t)$, and $d_{x_{2}}(t)$ are bounded, $\Delta f\left(x_{1}, x_{2}\right)$ and $d(t)$ are also bounded. From (20) to (22), it is clear that the synchronization problem of the two chaotic systems is replaced with a stabilization problem of the error dynamics.

To synchronize the chaotic gyros, the proposed RNDI controller is adopted in the response system in $40 \mathrm{sec}$. It is assumed that $\left|d_{x_{1}}(t)+\Delta f\left(x_{11}, x_{12}\right)\right| \leq 0.5$ and $\mid d_{x_{2}}(t)+$ $\Delta f\left(x_{21}, x_{22}\right) \mid \leq 0.7$. The initial conditions are $x_{11}(0)=1$, $x_{12}(0)=-1$, and $x_{21}(0)=1.6, x_{22}(0)=0.8$.

3.2. Simulation Results. In this section, numerical simulation results are provided in order to demonstrate the feasibility and effectiveness of the proposed RNDI controller.

Figures 7 and 8 illustrate the state and error trajectories of $\theta$ and $\dot{\theta}$ of the unsynchronized chaotic gyros, respectively; that is, no control action is performed in this case. The behaviors of the unsynchronized chaotic gyros oscillate independently due to the distinct initial conditions. Since there is no control action for synchronization, the errors of $\theta$ and $\dot{\theta}$ between the drive and response systems do not converge to zero as shown in Figures 7(b) and 8(b). Actually, the behaviors during $t=5-17$ sec seem to be synchronized in these figures. However, these are not the result of synchronization but are instead due to dynamic characteristics of the considered systems.

Figures 9 and 10 show the synchronized results of chaotic gyros under distinct initial conditions. In this case, the control input generated by the proposed RNDI controller forces the states of the response system to track those of the drive system. It is assumed that the RNDI controller is activated in $t=40 \mathrm{sec}$ to distinguish the dynamic behavior during $t=5-17 \mathrm{sec}$ from the synchronized behavior. Once the controller is activated in $t=40 \mathrm{sec}, \theta$ and $\dot{\theta}$ of the response system can track those of the drive system as shown in Figures 9(a) and 10(a). Moreover, the errors of $\theta$ and $\dot{\theta}$ between the drive and response systems converge to zero within a $1 \mathrm{sec}$ (Figures 9(b) and 10(b)). Hence, the proposed RNDI technique achieves the synchronization of chaotic systems containing uncertainties of model mismatches and disturbances under distinct initial conditions.

\section{Conclusion}

In this paper, a robust nonlinear dynamic inversion (RNDI) method has been proposed to solve the synchronization problem of chaotic gyros. The proposed RNDI improves robustness of NDI. Simulation results with synchronization of chaotic gyros using the proposed method show that accurate control performance can be achieved even in 


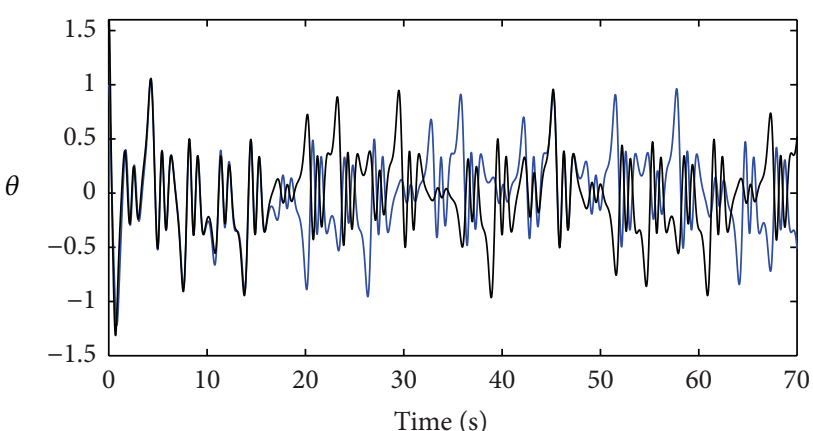

$\theta$ of the drive system
$\theta$ of the response system

(a)

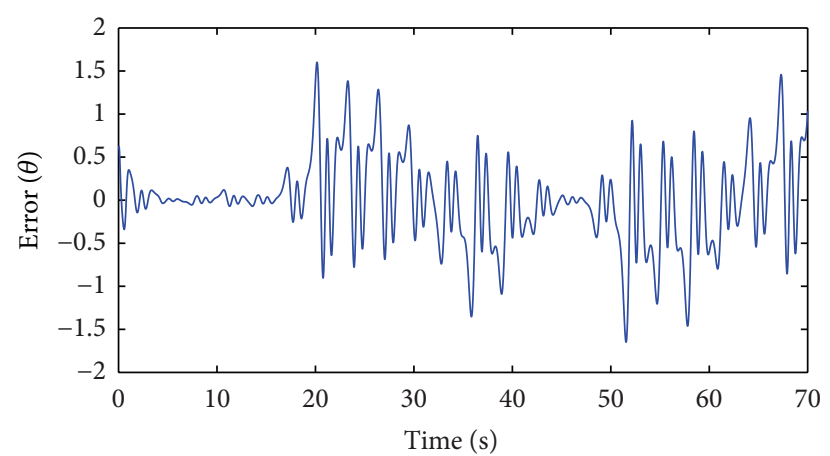

(b)

FIGURE 7: Simulation results of $\theta$ and error trajectories of unsynchronized chaotic gyros: (a) trajectories of $\theta$ of drive and response systems, (b) error behavior of $\theta$.

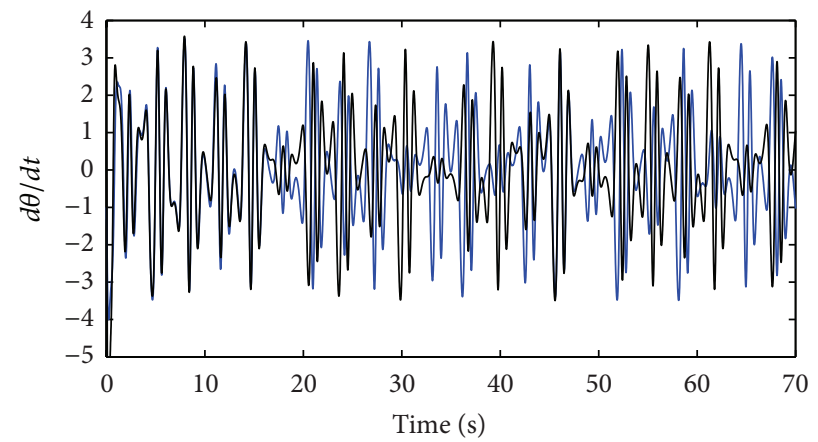

- $d \theta / d t$ of the drive system

— $d \theta / d t$ of the response system

(a)

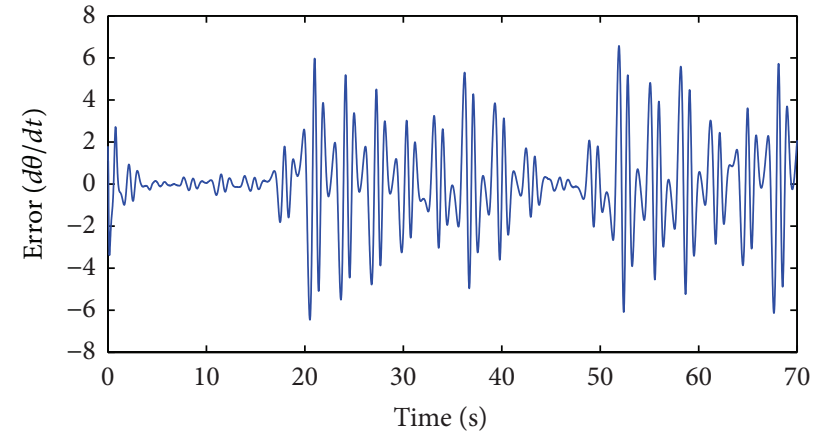

(b)

FIGURE 8: Simulation results of $\dot{\theta}$ and error trajectories of unsynchronized chaotic gyros: (a) trajectories of $\dot{\theta}$ of drive and response systems, (b) error behavior of $\dot{\theta}$.

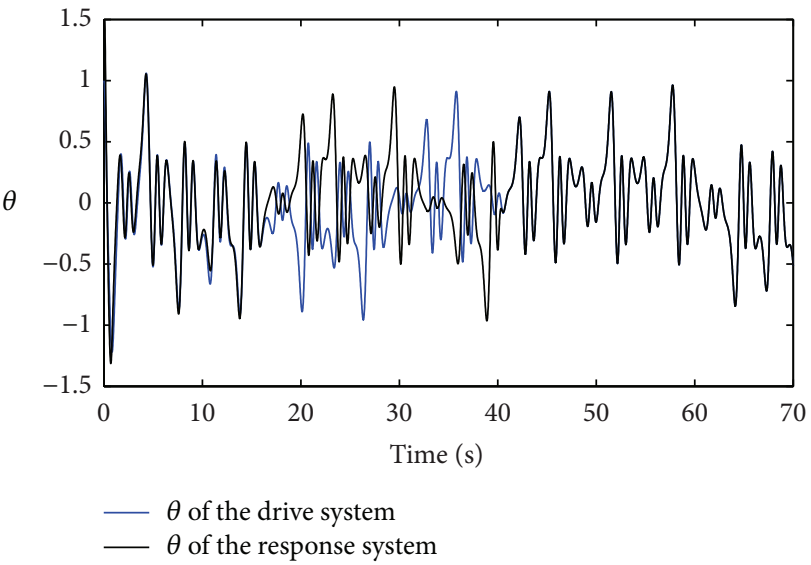

(a)

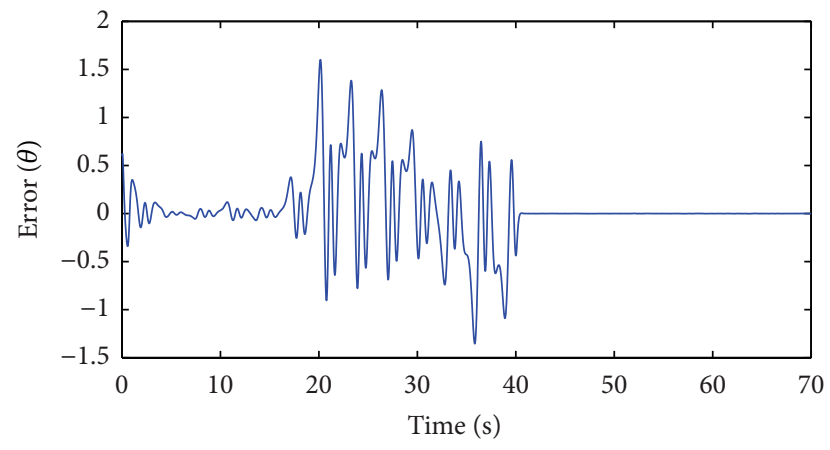

(b)

FiguRE 9: Simulation results of $\theta$ and error trajectories of synchronized chaotic gyros: (a) trajectories of $\theta$ of drive and response systems, (b) error behavior of $\theta$. 


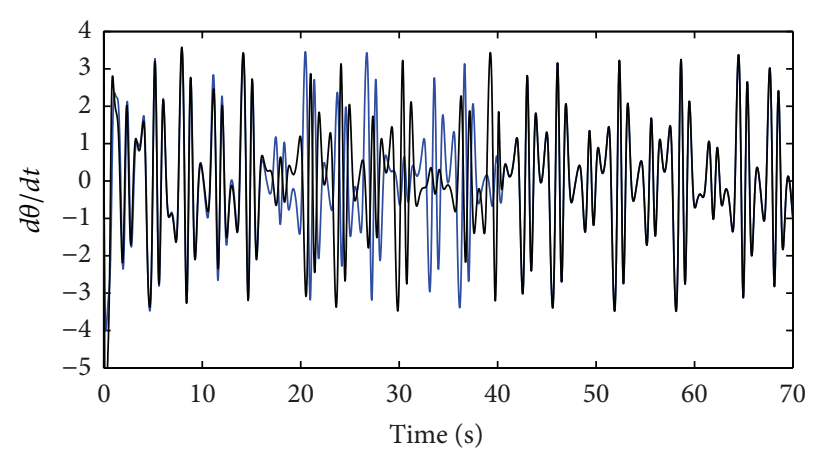

- $d \theta / d t$ of the drive system

— $d \theta / d t$ of the response system

(a)

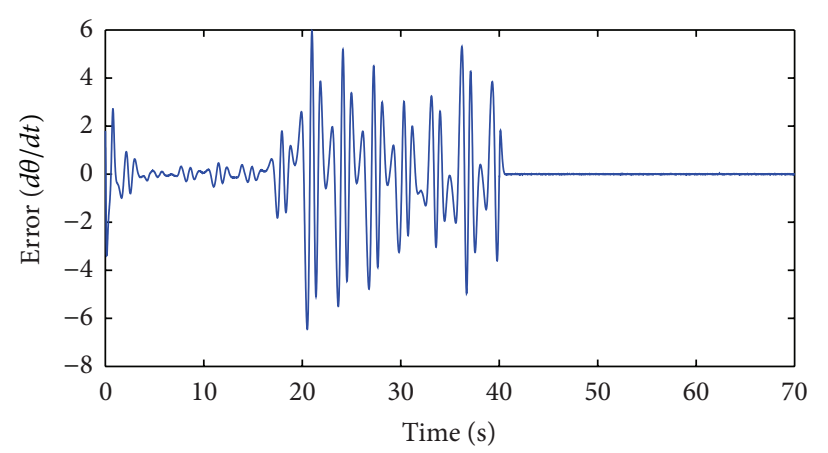

(b)

FIGURE 10: Simulation results of $\dot{\theta}$ and error trajectories of synchronized chaotic gyros: (a) trajectories of $\dot{\theta}$ of drive and response systems, (b) error behavior of $\dot{\theta}$.

the presence of uncertainties without any additional outerloop controller.

\section{Acknowledgment}

This research was supported by the the Ministry of Knowledge Economy (MKE), Republic of Korea, under the Convergence Information Technology Research Center (CITRC) support Program (NIPA-2013-H0401-13-1005) supervized by the National IT Industry Promotion Agency (NIPA).

\section{References}

[1] L. M. Pecora and T. L. Carroll, "Synchronization in chaotic systems," Physical Review Letters, vol. 64, no. 8, pp. 821-824, 1990.

[2] T. Liao and S. Tsai, "Adaptive synchronization of chaotic systems and its application to secure communications," Chaos, solitons and fractals, vol. 11, no. 9, pp. 1387-1396, 2000.

[3] F. Wang and C. Liu, "Synchronization of unified chaotic system based on passive control," Physica D, vol. 225, no. 1, pp. 55-60, 2007.

[4] X. Tan, J. Zhang, and Y. Yang, "Synchronizing chaotic systems using backstepping design," Chaos, Solitons \& Fractals, vol. 16, no. 1, pp. 37-45, 2003.

[5] B. A. Idowu, U. E. Vincent, and A. N. Njah, "Control and synchronization of chaos in nonlinear gyros via backstepping design," International Journal of Nonlinear Science, vol. 5, no. 1, pp. 11-19, 2008.

[6] J.-J. Yan, M.-L. Hung, and T.-L. Liao, "Adaptive sliding mode control for synchronization of chaotic gyros with fully unknown parameters," Journal of Sound and Vibration, vol. 298, no. 1-2, pp. 298-306, 2006.

[7] M. Jang, C. Chen, and C. Chen, "Sliding mode control of chaos in the cubic Chua's circuit system," International Journal of Bifurcation and Chaos in Applied Sciences and Engineering, vol. 12, no. 6, pp. 1437-1449, 2002.

[8] G. Chen and X. Dong, From Chaos to Order: Methodologies, Perspectives and Applications, vol. 24, World Scientific, Singapore, 1998.
[9] G. Chen, Control and Synchronization of Chaos: A Bibliography, Department of Electrical Engineering, University of Houston, Houston, Tex, USA, 1997.

[10] H.-K. Chen, "Chaos and chaos synchronization of a symmetric gyro with linear-plus-cubic damping," Journal of Sound and Vibration, vol. 255, no. 4, pp. 719-740, 2002.

[11] R. B. Leipnik and T. A. Newton, "Double strange attractors in rigid body motion with linear feedback control," Physics Letters A, vol. 86, no. 2, pp. 63-67, 1981.

[12] D. Ito, J. Georgie, J. Valasek, and D. T. Ward, "Reentry vehicle flight controls design guidelines: dynamic inversion," NASA/TP 2002-210771, 2002.

[13] D. Enns, D. Bugajski, R. Hendrick, and G. Stein, "Dynamic inversion: an evolving methodology for flight control design," International Journal of Control, vol. 59, pp. 71-91, 1994.

[14] I. Yang, D. Kim, and D. Lee, "A flight control strategy using robust dynamic inversion based on sliding mode control," AIAA 2012-4527, 2012.

[15] A. J. Ostroff and B. J. Bacon, "Force and moment approach for achievable dynamics using nonlinear dynamic inversion," AIAA 99-4001, 1999.

[16] R. J. Adams and S. S. Banda, "Robust flight control design using dynamic inversion and structured singular value synthesis," IEEE Transactions on Control Systems Technology, vol. 1, no. 2, pp. 80-92, 1993.

[17] H. Sira-Ramirez, "Differential geometric methods in variablestructure control," International Journal of Control, vol. 48, no. 4, pp. 1359-1390, 1988.

[18] R. A. DeCarlo, S. H. Zak, and G. P. Matthews, "Variable structure control of nonlinear multivariable systems: a tutorial," Proceedings of the IEEE, vol. 76, no. 3, pp. 212-232, 1988. 


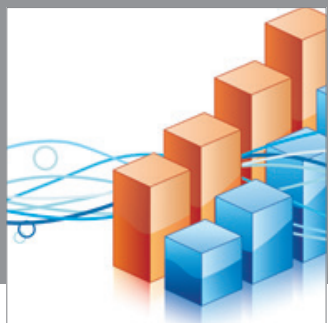

Advances in

Operations Research

mansans

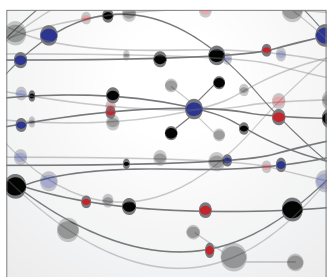

The Scientific World Journal
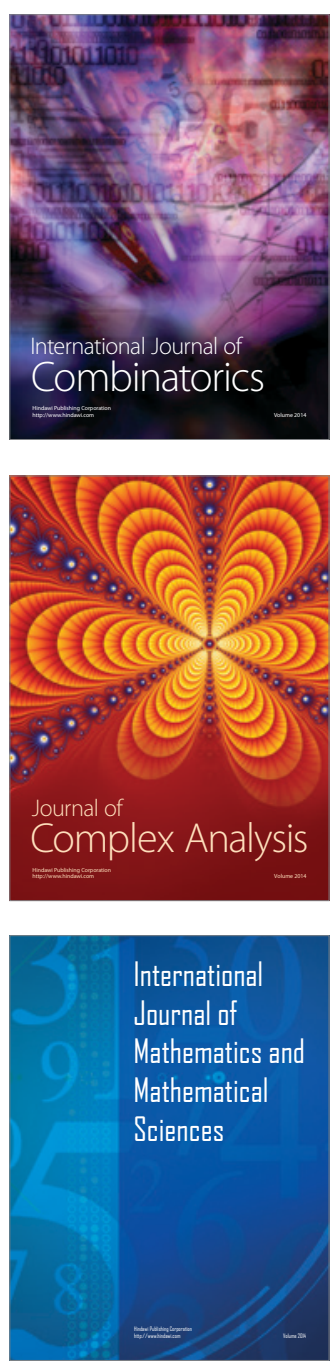
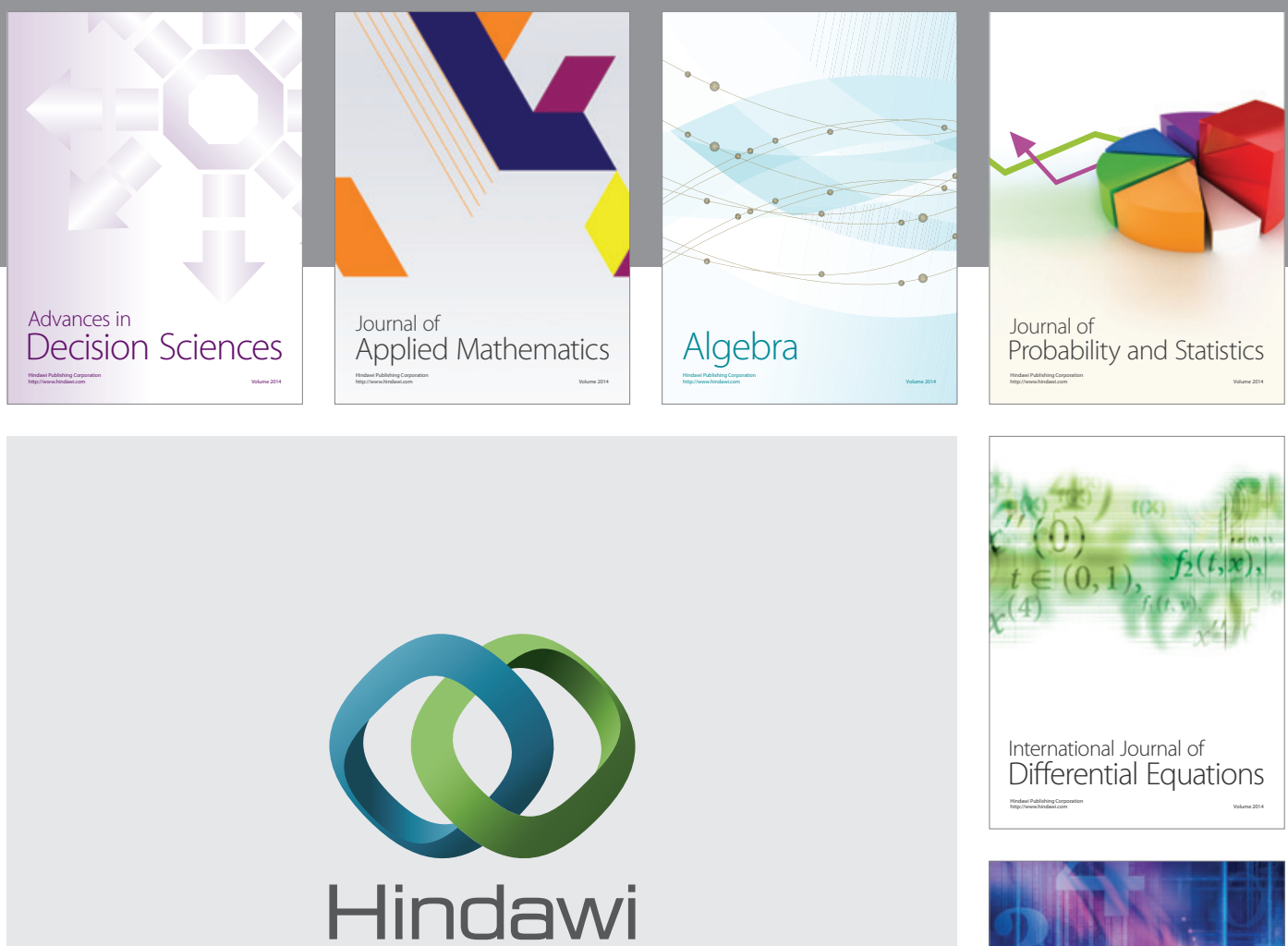

Submit your manuscripts at http://www.hindawi.com
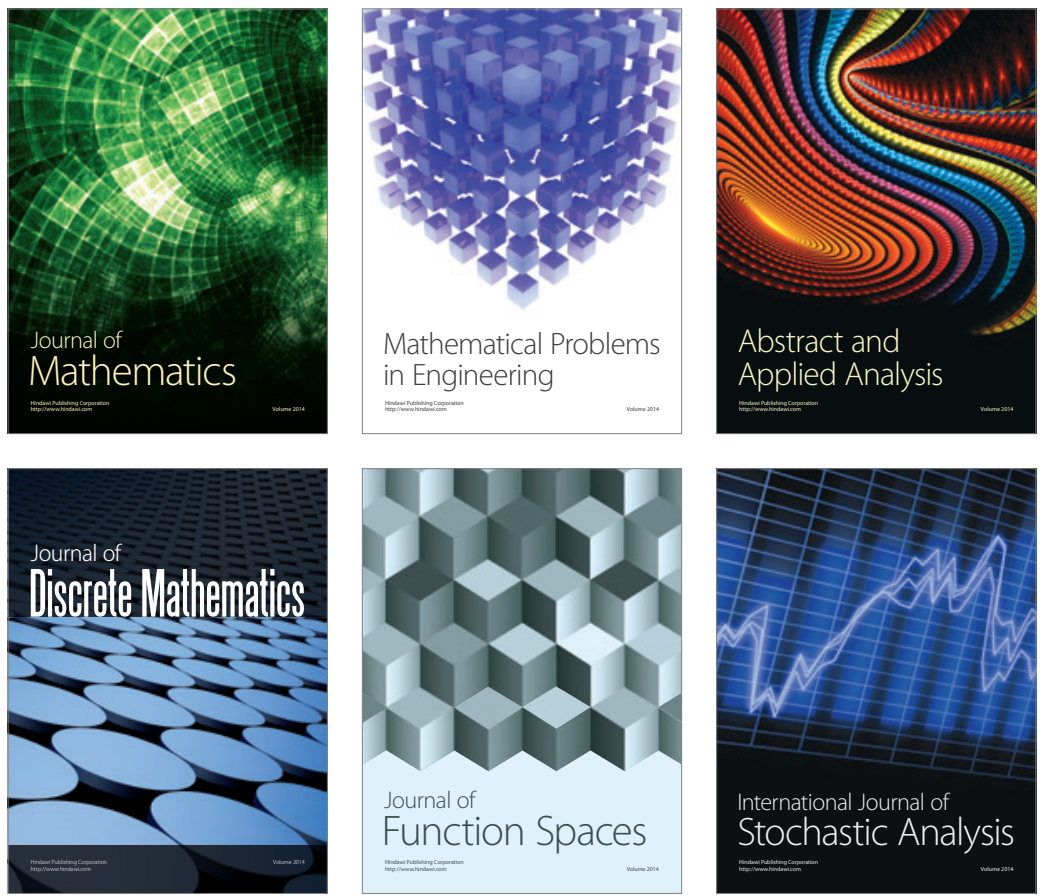

Journal of

Function Spaces

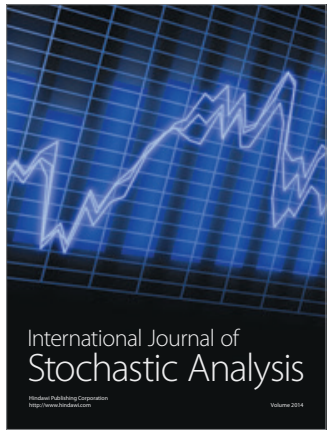

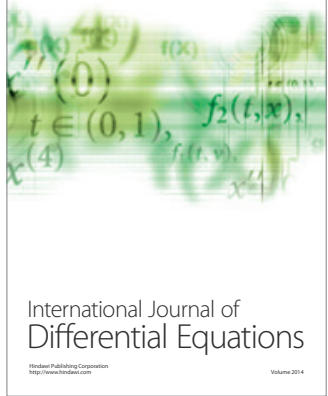
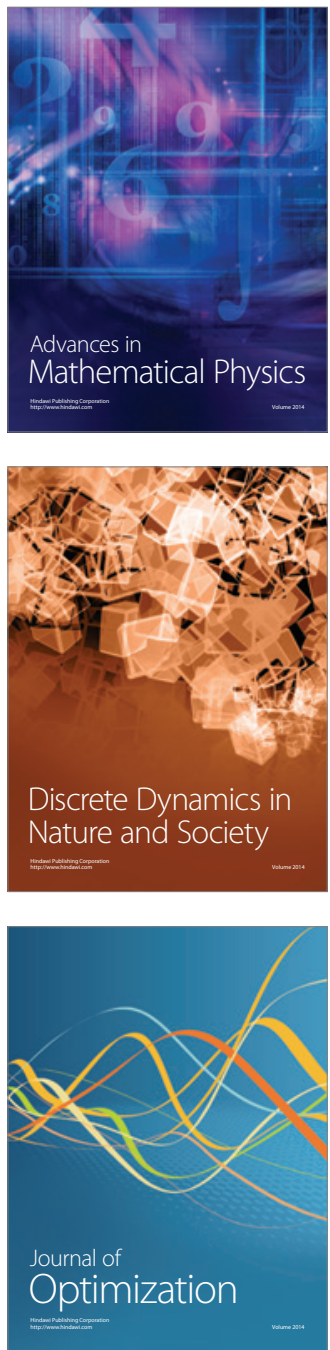